\title{
El docente frente a la innovación
}

Autoras:

Betsi Arechua ${ }^{4}$

vero8349@hotmail.com

Oleida Hurtado 5

oleidahurtado@hotmail.com

Irma Solar ${ }^{6}$

mashi_petto@hotmail.com

Recibido en noviembre de 2019, aceptado en diciembre de 2019

\section{Resumen}

Este artículo pretende compartir la experiencia vivida con docentes de básica media que formaron parte de un proceso de investigaciónacción-participativa enfocado en la innovación en el aula. En la Unidad Educativa Fe y Alegría ubicada en Santo Domingo de los Tsáchilas Ecuador, se percibía desinterés por aplicar estrategias innovadoras que actualmente se encuentran institucionalizadas en el centro, tras un largo proceso formativo al cuerpo docente.

Aquella situación motivó a un grupo de educadoras a indagar en sus causas, para posteriormente planificar y ejecutar acciones encaminadas a modificar la desidia ante las nuevas propuestas educativas. De allí surge un breve proceso formativo impartido por docentes y dirigido a otras

$4 \quad$ Licencia en ciencias de la educación mención educación primaria.

Educadora en la básica elemental y media.

5 Profesora en educación primaria. Licenciada en ciencias de la educación. Educadora en la básica elemental y media. Ex directora de la Unidad Educativa Fe y Alegría Santo Domingo. Se ha desempeñado como profesora unidocente en la escuela Condorazo Fe y Alegría Manabí.

6 Intérprete de Lengua de Señas Ecuatorianas certificada por FENASEC y SECAP. Candidata a: licenciada en educación por la Universidad de Especialidades Espíritu Santo. Intérprete de LSEC, educadora en preparatoria, básica elemental y básica media en la Unidad Educativa Fe y Alegría Santo Domingo. 
compañeras y compañeros docentes titulado: Libro Rojo. Dicho esfuerzo permitió fortalecer la planificación curricular en: estrategias, destrezas y rutinas para innovar en el proceso de enseñanza - aprendizaje.

Palabras clave: Innovación educativa, formación docente, metodologías activas, investigación acción participativa, educadores investigadores

\section{The teacher in the face of innovation}

\section{Abstract:}

This article is aimed to share the experience with teachers of elementary school that were part of the participative action research process focused on innovation in the classroom. There is a lack of interest in applying innovative strategies that are currently institutionalized in Unidad Educativa Fe y Alegría located in Santo Domingo de los Tsáchilas - Ecuador, after a lengthy training process for the teaching staff.

A group of teachers undertook the situation analysis to find the causes, to later plan and carry out actions aimed at modifying the indifference of the new educational proposals. Consequently, a training process grows. Teachers taught it and aimed at other fellow teachers: Red Book. That effort brought a strengthening curricular planning in: strategies, skills and routines to innovate in the teaching-learning process.

Keywords: Educational innovation, teacher training, active methodologies, participative action research, research teachers.

\section{Introducción}

"La formación docente tiene el honor de ser, simultáneamente, el peor problema y la mejor solución en educación" (Aguerrondo, 2004, p. 97 en Fullan, 1993).

Los docentes en su accionar cotidiano pueden llegar a reproducir prácticas rutinarias que se corresponden con una actitud pasiva frente 
a su rol. La rutinización de la práctica educativa puede configurar en el docente cierta resistencia a salir de su zona de confort; este hecho se muestra con mayor fuerza cuando el educador ha enseñado, por varios años, los mismos temas y lo ha venido haciendo de la misma forma. Persiguiendo la revalorización y mejora del ejercicio docente, Fe y Alegría organización a la que pertenece el centro educativo donde se ha llevado a cabo la investigación que el presente artículo aborda, ha implementado, desde hace algunos años, una serie de talleres de formación con miras a responder a las nuevas necesidades del educando actual.

A partir de este proceso formativo se esperaba tener como resultado maestros(as) motivados y deseosos de aplicar las nuevas metodologías y estrategias aprendidas, sin embargo, en la Unidad Educativa Fe y Alegría de Santo Domingo (Ecuador) persiste la monotonía y falta de interés por parte de algunos educadores y educadoras. Por otro lado, puede identificarse que los docentes no aplican de manera continua las estrategias metodológicas institucionalizadas al momento de impartir la clase y utilizan poco o nada los recursos y herramientas tecnológicas.

Al interior del centro, concretamente en el subnivel medio donde se centra el estudio, el desinterés mostrado por algunos docentes respecto a la aplicación de estrategias innovadoras ha influido en el desarrollo de la clase y en el rol asumido por los estudiantes, quienes tienen limitadas posibilidades de desempeñarse como agentes del proceso de enseñanza aprendizaje. La limitada aplicación de estrategias innovadoras ha repercutido en el desarrollo cognitivo de las y los estudiantes, hecho que se venía evidenciando en las juntas de cursos, donde los docentes manifestaron en diversas ocasiones que el alumnado tiene un bajo rendimiento académico y disciplinario. Por otro lado, la dimensión pedagógica, encargada de revisar y analizar todos los documentos pedagógicos presentados por el profesorado, sostiene que muchos de ellos no se encuentran animados a utilizar las Tics, y muestran una apertura limitada para impartir sus clases bajo el uso de herramientas distintas a las acostumbradas.

7 Fe y Alegría es un Movimiento de Educación Popular y Promoción Social dirigido a sectores empobrecidos y excluidos con el fin de potenciar sus capacidades personales y su desarrollo y participación en la sociedad. 
Con el propósito de dar respuesta a esta dificultad un equipo de docentes se valió de un proceso investigativo que se desarrolló a lo largo de los años 2017 y 2018, el cual se orientó a buscar alternativas que motiven un cambio en la actitud del profesorado. La finalidad de este artículo es dar a conocer los resultados obtenidos en dicho proceso, mismo que adoptó el enfoque de investigación acción participativa (IAP), lo que supuso que, además de indagar, el equipo de educadoras investigadoras debía proponer un plan de acción a partir de los hallazgos. En línea con el enfoque de investigación seleccionado, el documento pretende mostrar una síntesis de las acciones implementadas con el fin de reflexionar la práctica diaria del docente, la importancia de utilizar las diferentes estrategias metodológicas implementadas en la institución y el impacto de las estrategias innovadoras en la planificación y el desarrollo de alumnos(as) más críticos y reflexivos.

\section{Metodología}

La investigación acción participativa prioriza la construcción de conocimiento a partir del diálogo con quienes construyen la realidad. La naturaleza participativa y el carácter colaborativo de la investigaciónacción lo explica Kemmis \& McTaggart (1988):

...una forma de indagación introspectiva colectiva emprendida por participantes en situaciones sociales con objeto de mejorar la racionalidad y la justicia de sus prácticas sociales o educativas, así como su comprensión de esas prácticas y de las situaciones en que éstas tienen lugar. (Kemmis \& McTaggart, p.9)

Para el desarrollo de la investigación se realizaron entrevistas a docentes de la básica media con el fin de obtener información sobre las metodologías y estrategias incluidas o no en la planificación, y las razones por las que éstas no estaban reflejadas en los documentos entregados y revisados por la dimensión pedagógica del centro educativo.

Posteriormente se convocó a una mesa redonda ${ }^{8}$ con las autoridades del plantel, autoridades de la oficina zonal de Fe y Alegría (entidad encargada de acompañar, formar y dar seguimiento a los

8 Debate entre varios expertos para tratar sobre un asunto específico. 
procesos impulsados por la organización en los centros educativos del sector), docentes de la básica media y el equipo de investigación, lo que permitió obtener información relevante para contrastar diferentes criterios. Se elaboró una encuesta aplicada a estudiantes y docentes de la básica media, instrumento que integró preguntas abiertas y cerradas las cuales otorgaron datos adicionales que contribuyeron a la planificación y ejecución del plan de acción.

A partir de los resultados de los distintos instrumentos se implementó el plan de acción, el cual consistió en la ejecución de varios talleres de estrategias pedagógicas impartidos por el equipo investigador y dirigidos a los y las docentes interesadas en la propuesta.

\section{Resultados}

\section{Etapa de investigación: presentación de los resultados más relevantes}

En la etapa de la investigación, mediante la aplicación de encuestas dirigidas a docentes y estudiantes de la básica media (quinto, sexto, séptimo de educación general básica), se evidenció que: el $57 \%$ del alumnado encuestado expresó que los tutores no aplicaban las estrategias institucionalizadas frente al $43 \%$ que aduce que sí las utilizaban. Por su parte, los resultados de la encuesta a los docentes muestran que el $70 \%$ no utiliza las estrategias innovadoras porque no tienen tiempo, dado que deben responder a varios procesos simultáneos, mientras que el $30 \%$ restante indica que sí las aplica. La encuesta fue aplicada a un total de 7 docentes y a 361 estudiantes. Ver Tabla 1.

Tabla $\mathbf{N}^{\circ}$ 1: Resultados encuesta a docentes

\begin{tabular}{|l|c|c|}
\hline & $\begin{array}{l}\text { Encuesta aplicada a } \\
\text { docentes }\end{array}$ & $\begin{array}{l}\text { Encuesta aplicada a } \\
\text { estudiantes }\end{array}$ \\
\hline $\begin{array}{l}\text { Indica que no aplica estrate- } \\
\text { gias innovadoras }\end{array}$ & $70 \%$ & $57 \%$ \\
\hline $\begin{array}{l}\text { Indica que aplica estrategias } \\
\text { innovadoras }\end{array}$ & $30 \%$ & $43 \%$ \\
\hline
\end{tabular}


Con respecto a la mesa de diálogo que giró en torno al tema: causas de la no aplicación de estrategias innovadoras implementadas en el centro educativo y los pasos para las posibles soluciones, los docentes alegaron que no aplican estas estrategias dada la saturación de trabajo, ya que desde Fe y Alegría se exigen resultados de diferentes procesos y el docente debe responder a todos ellos a la par, además del exceso de estudiantes en cada aula y el reducido tiempo que se otorga para efectuar cada estrategia. Los directivos, por el contrario, expresaron que se ha dado el tiempo necesario para la aplicación de estrategias y que el número de estudiantes corresponde a lo permitido por el Ministerio de Educación de Ecuador. Aquello demuestra una confrontación en las posturas de los actores involucrados, lo que pudiese ser parte de las causales del problema en cuestión.

Sin embargo, tanto docentes como directivos reconocieron que la no aplicación de estrategias está relacionada con una comprensión limitada de las mismas y con una baja motivación para prepararse de manera autónoma y continua. Este acuerdo permitió vislumbrar algunos de los aspectos que debían ser reforzados: conocimiento respecto a las estrategias y motivación.

\section{Etapa de ejecución del plan de acción}

El plan de acción, que fue la etapa posterior al proceso investigativo, buscó concretar acciones que contrarresten las dos dificultades visualizadas con mayor claridad en la etapa diagnóstica. Se propuso, por un lado, dictar talleres pedagógicos que abarcaron desde la malla curricular hasta las estrategias de innovación educativa y, por otro, se planteó la incorporación de un equipo encargado de buscar espacios una vez al mes para la recreación y fomento de un ambiente de interrelación social. Ambas acciones estuvieron propuestas para docentes y administrativos.

Es importante resaltar que, si bien ambas actividades fueron planteadas a las autoridades del centro, solo una de ellas pudo ser implementada: los talleres pedagógicos. La propuesta encaminada a generar espacios que mejoren las relaciones interpersonales y el clima laboral como medio para motivar al profesorado no se llevó a cabo 
ya que los directivos de ambas jornadas de la institución (matutina y vespertina) no llegaron a un consenso para su implementación, pues no encontraron los horarios adecuados para beneficiar a ambas jornadas sin perjudicar el tiempo pedagógico.

Para la ejecución de los talleres pedagógicos el equipo de investigadoras realizó inicialmente una campaña de expectativa la cual despertó curiosidad en las y los docentes. Con algunos días de anticipación se entregó a los educadores y educadoras una invitación poco convencional que llamara su atención y, llegado el día, el salón en el que se explicó al profesorado la iniciativa fue decorado de manera llamativa utilizando el color rojo, característico de esta propuesta de formación a la cual se la denominó: El Libro Rojo. El día de socialización de la propuesta fue determinante en el proceso, ya que la intencionalidad era que las y los docentes se sintieran atraídos por la propuesta para que decidieran acogerla, pese a las variadas actividades y formaciones de diversa índole que realizan cotidianamente. Algunas de las acciones realizadas ese día fueron colocar un libro rojo, el cual no podía ser abierto e invitar a las personas a ingresar al espacio con los ojos vendados para generar expectativa.

Aquel día se explicó la modalidad de los talleres, se establecieron cupos limitados y, cumpliendo con los objetivos propuestos, se logró la inscripción de la totalidad de los cupos previstos (7 docentes). El proceso inició de inmediato, durante la primera reunión, que se llevó a cabo el mismo día de la socialización e inscripción, se entregó a las participantes un folleto rojo donde cada maestra registraría apuntes a lo largo de todos los talleres, aquel folleto se convirtió en el símbolo del plan de acción.

Un logro relevante fue la asistencia constante a cada uno de los talleres aun cuando fueron realizados en horarios extra laborales. Se planificó coordinadamente desde la oficina del vicerrectorado las fechas y horarios para ejecutar las actividades formativas dentro de los cronogramas parciales, lo que permitió que se desarrollaran de manera organizada. El proceso se extendió a lo largo de 3 meses con intervalos de 15 días entre cada taller. En este periodo las docentes se fortalecieron 
en: currículo, CORDIS ${ }^{9}$, paleta de inteligencias múltiples (I.M.) ${ }^{10}$, rutinas y destrezas de pensamiento, rúbrica y lista de cotejo. Dichos temas fueron seleccionados por las participantes, fueron escogidos colectivamente de acuerdo a las debilidades existentes.

Otra de las metas propuestas para este proceso fue que la formación no se quedase únicamente en las 7 personas que la recibieron. Aquel objetivo se alcanzó, pues todas las docentes aceptaron el reto de replicar con otras educadoras y educadores lo aprendido y ponerlo en práctica en sus aulas. Quizás uno de los logros más importantes fue evidenciar mayor autoconfianza en el desarrollo de sus planificaciones microcurriculares utilizando diferentes estrategias. Cabe resaltar, además, que el equipo directivo se comprometió a seguir brindando espacios dentro de los cronogramas quimestrales para la formación docente.

Al finalizar El Libro Rojo el equipo de investigadoras realizó una evaluación práctica mediante la cual se pidió a las educadoras elaborar una planificación con las estrategias afianzadas en los talleres. El resultado demostró que el $89 \%$ de las educadoras contemplaron en sus planificaciones elementos suficientes para desarrollar su práctica utilizando lo aprendido en las formaciones, mientras que el $11 \%$ alcanzó un nivel deseable. Las evaluaciones, por ende, evidenciaron que las docentes lograron aprendizajes y despejaron dudas en varias estrategias para poder plasmarlas en planificaciones que respondan a sus contextos e incluyan actividades innovadoras.

Este proceso permite reconocer la importancia de la formación entre pares, puesto que ayuda a visualizar debilidades,

9 Contextualización (CO), Revalorización de saberes y experiencias (R), Diálogo de saberes D), Innovación transformadora (I), Sistematización y socialización. El CORDIS es el Sistema metodológico de Fe y Alegría.

10 Howard Gardner, en su investigación plantea la existencia de ocho formas para aprender, unas más desarrolladas que otras, según las personas. De este modo, los individuos son capaces de conocer el mundo por medio del lenguaje, del análisis lógico-matemático, de la representación espacial, el pensamiento musical, el uso del cuerpo para resolver problemas o hacer cosas, de una comprensión de los demás individuos y de nosotros, así como del contacto con el medio que los rodea (Paniagua, K. 2008). 
a reforzar aprendizajes y a mantener una formación continua que no dependa de personas externas. Es una necesidad del docente estar en formación constante para responder al contexto cambiante en el que se desenvuelve $y$, en este sentido, resulta imperativo reconocer la comunicación horizontal y la formación entre colegas como un medio para revalorizar los saberes de educadores y educadoras y permitir un intercambio fructífero al interior de los centros educativos.

\section{Reflexiones del proceso:}

Es necesario que el docente vea la necesidad de ampliar sus conocimientos, profundizar en lo aprendido y mejorar como profesional, sin embargo, "la formación docente tiene el honor de ser, simultáneamente, el peor problema y la mejor solución en educación" (Aguerrondo, 2004, p. 97 en Fullan, 1993). Aunque, la formación dentro del imaginario colectivo no suele ser percibida como un problema en sí mismo, el ritmo del docente, sus múltiples labores tanto pedagógicas como administrativas reducen los tiempos y espacios para actividades de esta índole. Aquello se hace evidente en la Unidad Educativa Fe y Alegría Santo Domingo donde las y los docentes concordaron en no tener el tiempo suficiente para apropiarse de las estrategias de aula impartidas a lo largo de un extenso proceso formativo, puesto que sus horas extracurriculares y extra laborales son ocupadas por varios procesos de Fe y Alegría y ministeriales que deben ser atendidos de manera permanente.

La carga laboral, por ende, podría ser una de las causas que repercuten en la desmotivación de las educadoras y los educadores. No obstante, Spark "indica que la resistencia al cambio es sólo un síntoma de la baja calidad de la innovación propuesta y la falta de respeto a la experiencia previa de los propios profesores" (Paredes Labra, 2004, p.136). Este planteamiento nos lleva a pensar que la raíz de la desmotivación podría residir también en inadecuados modos de plantear los procesos de innovación educativa y posiblemente a una desvalorización de la experiencia docente. Desde la experiencia analizada en el presente artículo se vislumbra que tras la formación recibida varias estrategias de innovación debían ser aplicadas en el aula, pero por falta de interés, motivación y conocimiento del 
docente no eran replicadas, por ende, sus clases seguían siendo monótonas.

Muchas veces confunde pensar que el profesor es solo el ejecutor de la innovación, y no es así; el profesor es el encargado de mediar dicha innovación, leerá, comprenderá, asimilará y redefinirá el cambio a darse a partir del contexto. El profesor es el agente principal de la innovación. (Sánchez Asín, Boix y Jurado, 2009). Entendida de este modo la innovación, el proceso formativo no supone la recepción de contenidos y metodologías por parte de externos para ser replicadas en las aulas, sino un proceso de redefinición de los nuevos contenidos a partir del contexto específico y la experiencia previa del docente.

En esta línea la experiencia vivida, que parte de una formación que no fue asimilada adecuadamente por la totalidad de las y los docentes, pero que tras una formación complementaria entre pares logra mejores resultados da nuevas luces sobre alternativas formativas que puedan ser recibidas con mayor apertura por el profesorado. La experiencia radica en una formación de docentes a docente, es decir, es un trabajo colaborativo entre educadores en el que el profesor debe desarrollar destrezas sociales.

Al respecto, Rudduck en referencia al desarrollo profesional del docente decía que, es la capacidad de un profesor para mantener la curiosidad acerca de la clase; identificar intereses significativos en el proceso de enseñanza y aprendizaje; valorar y buscar el diálogo con colegas expertos". (Marcelo, 2009 p.43 en Rudduck, 1991)

Siguiendo este lineamiento el desarrollo profesional del docente debe basarse en el diálogo entre pares y en una investigación permanente. Asimismo,

Miller y Peterson comentan acerca de la gran variedad de situaciones que se encuentran bajo el epígrafe de "intervenciones pedagógicas basadas en las relaciones entre iguales" (...) Estassituaciones suelen estar planificadas por algún miembro con responsabilidad, con el fin de alcanzar tanto objetivos académicos como afectivos. (Samaniego, 1995, p.181) 
Situación que como se ha manifestado previamente resultó fructífera en la presente experiencia. En la unidad educativa las participantes de los talleres del Libro Rojo mencionaron que al recibir la formación por parte de sus compañeras, quienes se encuentran en el mismo contexto laboral, sentían mayor confianza y se mostraban más receptivas.

Resulta interesante haber evidenciado incluso que, pese a la carga laboral descrita previamente, las participantes del Libro Rojo demostraron una actitud positiva al dar tiempos extra para poder cumplir y completar el cronograma establecido para la formación. La preocupación por los ajustados tiempos y múltiples labores era evidente, mas no desmotivadora, aun cuando en varias ocasiones los horarios hacían más extensa su jornada laboral.

Cabe resaltar, que hubo otros elementos que pudieron contribuir a un desarrollo favorable de esta formación. Uno de ellos fue la no imposición de las temáticas a ser abordadas, puesto que éstas fueron elegidas por las docentes participantes. El equipo de investigación tenía pensada una estructura distinta de los talleres a la que finalmente se ejecutó acogiendo las peticiones de las docentes. Otro elemento relevante fue el limitar el número de cupos para el proceso, ya que desde un inicio se pensó que la enseñanza debía ser personalizada para poder obtener resultados de calidad y calidez generando espacios de confianza a corto plazo.

Sin duda, el reducido número de participantes hizo posible que la comunicación sea más cercana. Propiciar espacios de encuentro con esta característica resultó favorable para propiciar un clima de trabajo armonioso. Como lo señala Jares (2006) es necesario potenciar las relaciones que favorezcan el afecto, estima y seguridad en los grupos, contribuir a la definición de metas grupales conocidas y compartidas, motivar una comunicación de calidad, promover el sentido de pertenencia y cuidar los procesos de vida de las personas que integran los equipos de trabajo.

En definitiva, el mayor aprendizaje de esta investigación acción - participativa fue la verificación de que las comunidades de 
aprendizaje entre educadoras y educadores tiene efectos positivos. Se demostró que entre docentes existe más confianza para compartir conocimientos y despejar dudas, ya que se comparte la misma realidad dentro del centro. Fue gratificante también el compromiso espontaneo de cada participante del Libro Rojo por replicar a los demás docentes sus aprendizajes y se fortalecieron las relaciones interpersonales.

\section{Conclusiones}

El presente análisis deja algunas ideas claras. Por un lado, se evidenció que las y los docentes están conscientes de la baja apropiación y compromiso por parte del profesorado respecto al uso de las estrategias innovadoras. Asimismo, resultó notoria la inconformidad inicial en cuanto al exceso de trabajo que supone responder a los distintos procesos del centro. Un hallazgo que debe ser tomado en consideración es la necesidad de establecer tiempos amplios para la asimilación de los nuevos conocimientos pedagógicos, lo que implica además, que después de cada capacitación, su contenido sea replicado para afianzarlo y despejar dudas respecto a cómo y cuándo aplicarlo.

No obstante, quizás la constatación más relevante es la importancia de generar espacios en los que se dinamice la formación entre pares y que ésta conste dentro de las planificaciones de los centros educativos. La experiencia demostró que el grupo de docentes que participó del taller dictado por educadoras de su mismo centro se sintió en confianza, pues pudieron expresar dudas con mayor libertad y el trabajo se realizó con entusiasmo. Además, estos espacios permitieron afianzar el compañerismo, lo que permite deducir que el aprendizaje entre pares favorece no solo la adquisición de conocimientos, sino que contribuye a mejorar las relaciones entre docentes. Por último, se constató que dicha dinámica es capaz de despertar mayor compromiso por parte del profesorado, puesto que, aun cuando en reiteradas ocasiones se trabajó los talleres en horas extralaborales, aquello no fue un impedimento para su adecuado desarrollo y no mermó el interés y participación de las personas involucradas. Cabe recalcar, que uno de los aspectos que contribuyó a esta experiencia fue la motivación y expectativa generada al inicio del proceso, ya que ésta presentó a la 
formación como algo distinto a lo habitual y resultó llamativo para las educadoras.

\section{Referencias bibliográficas}

Aguerrondo, I. (2004). Los desafíos de la política educativa relativos a las reformas de la formación docente. AAVV. Maestros en América latina: Nuevas perspectivas sobre su formación y desempeño. Santiago de Chile: PREAL-CINDE, 97-142

Jares, X. R. (2006). Pedagogía de la convivencia (Vol. 228). Graó.

Kemmis, S. \& McTaggart, R. (1988). Cómo planificar la InvestigaciónAcción. Barcelona, España: Laertes.

Labra, J. P. (2004). Cultura escolar y resistencia al cambio. Tendencias pedagógicas, (9), 131-142.

Marcelo, C. (2009). La evaluación del desarrollo profesional docente: de la cantidad a la calidad. Revista Brasileira de Formação de professores, 1(1), 43-70.

Sánchez, A., Boix, J. L. \& Jurado, P. (2009): La sociedad del conocimiento y las TICs: Una inmejorable oportunidad par el cambio. Pixel Bit. Revista de Medios y Educación, 34, 179- 205

Samaniego, M. C. M. (1995). La interacción entre compañeros: el conflicto sociocognitivo, el aprendizaje cooperativo y la tutoría entre iguales. Revista interuniversitaria de formación del profesorado, (23), 177-186. 